\title{
Numerical Solution of Nonlinear Stochastic Itô-Volterra Integral Equations Driven by Fractional Brownian Motion Using Block Pulse Functions
}

\author{
Mengting Deng $(\mathbb{D}$, Guo Jiang $\mathbb{D}$, and Ting Ke $\mathbb{D}$ \\ School of Mathematics and Statistics, Hubei Normal University, Huangshi 435002, Hubei, China \\ Correspondence should be addressed to Guo Jiang; gjiang@hbnu.edu.cn
}

Received 25 May 2021; Revised 7 September 2021; Accepted 30 September 2021; Published 30 October 2021

Academic Editor: Shiping Wen

Copyright (c) 2021 Mengting Deng et al. This is an open access article distributed under the Creative Commons Attribution License, which permits unrestricted use, distribution, and reproduction in any medium, provided the original work is properly cited.

\begin{abstract}
This paper presents a valid numerical method to solve nonlinear stochastic Itô-Volterra integral equations (SIVIEs) driven by fractional Brownian motion (FBM) with Hurst parameter $H \in((1 / 2), 1)$. On the basis of FBM and block pulse functions (BPFs), a new stochastic operational matrix is proposed. The nonlinear stochastic integral equation is converted into a nonlinear algebraic equation by this method. Furthermore, error analysis is given by the pathwise approach. Finally, two numerical examples exhibit the validity and accuracy of the approach.
\end{abstract}

\section{Introduction}

Stochastic equations have been widely used in engineering, economic management, biological sciences, finance, etc. A lot of problems in these areas are modeled by stochastic Volterra integral equations. However, the vast majority of these equations do not have exact solutions, so it is meaningful to discuss numerical solutions of the equations $[1,2,3,4,5,6,7]$.

In the present work, we use BPFs to solve the following nonlinear SIVIEs driven by FBM:

$$
x(u)=x_{0}(u)+\int_{0}^{u} g_{1}(s, u) v(x(s)) \mathrm{d} s+\int_{0}^{u} g_{2}(s, u) \kappa(x(s)) \mathrm{d} B_{s}^{H}, \quad u \in[0,1)
$$

where $g_{1}(s, u)$ and $g_{2}(s, u)$ are kernel functions, for $s \in[0, u) . x(u)$ is an unknown stochastic process, $B_{s}^{H}$ is FBM with $H \in((1 / 2), 1)$, and the stochastic integral is pathwise Riemann-Stieltjes (R-S) integral. They are all defined on the same probability space $(\Omega, \mathscr{F}, P) . \nu$ and $\kappa$ are bounded analytic functions and satisfy Lipschitz conditions.

Although FBM is not semimartingale or Markov process, FBM can describe more phenomena than Brownian motion. This is also the main reason why FBM can be used in the Internet, traffic, weather, and so on. In the past two decades, many scholars have extensively researched the stochastic equations driven by FBM [8-16]. According to [15], we only discuss the case where $H \in((1 / 2), 1)$, and the stochastic integral in equation (1) is the pathwise R-S integral. In [17], Zähle used the fractional integration by parts formula to express the pathwise R-S integral in terms of fractional derivative operators. In [18], Nualart and Răşcanu used the pathwise R-S integral to study stochastic differential equations in regard to FBM. Pei and Xu [19] solved the stochastic equations about FBM with $H>(1 / 2)$ and standard Brownian motion by pathwise approach and stochastic average. Moreover, Mishura and Shevchenko studied the Euler 
approximate solutions of stochastic differential equations in regard to FBM in [10]. Hu et al. [20] also gave the Euler scheme of stochastic differential equation with respect to FBM.

Since FBM is not martingale, the classical Itô formula is invalid. Hashemi and Khodabin [8] used Hat functions to solve nonlinear SIVIEs driven by FBM, but the error analysis is insufficient. On the contrary, Maleknejad et al. [3, 4], Sang et al. [6], and Ezzati et al. [12] studied numerical solution of stochastic equation using BPFs and gave the error analysis. In this paper, we employ BPFs to solve equation (1) and a high-precision numerical solution is acquired. In [10], authors prove that the rate of convergence for Euler approximations of solutions of pathwise SDEs driven by FBM with $H>(1 / 2)$ can be estimated by $O\left(\delta^{2 H-1}\right)$ ( $\delta$ is the diameter of partition). Compared with [10], the convergence rate of the approximate solution obtained by this method may be faster when $H \longrightarrow 1$. In addition, the calculation also is simpler.

This article's structure is as follows. In Section 2, the definitions and related properties of FBM and BPFs are given. In Section 3, the effective numerical method is used to solve equation (1). The error analysis is acquired in Section 4. In Section 5, the validity and accuracy of this approach are validated by two examples. Section 6 is the conclusion.

\section{Preliminaries}

FBM and BPFs have been extensively studied by many scholars. For more contents, please refer to $[3,4,14,16]$.

2.1. Fractional Brownian Motion. FBM is a stochastic process $\left(B_{u}^{H}, u \geq 0\right)$ with $H \in((1 / 2), 1)$ on $(\Omega, \mathscr{F}, P)$, and $B_{u}^{H}$ is a continuous Gaussian process that has zero mean and the following covariance function:

$$
\operatorname{Cov}\left(B_{s}^{H}, B_{u}^{H}\right)=\frac{1}{2}\left\{s^{2 H}+u^{2 H}-|u-s|^{2 H}\right\} .
$$

It satisfies the following properties:

(i) $B_{0}^{H}=0$ and $\mathbb{E}\left(B_{u}^{H}\right)=0, u \geq 0$

(ii) $B_{u}^{H}$ has homogeneous increments

(iii) $\mathbb{E}\left(B_{u}^{H}\right)^{2}=u^{2 H}, u \geq 0$

(iv) $B_{u}^{H}$ has continuous trajectories

It is obvious that $B_{u}^{H}$ is a standard Brownian motion when $H=(1 / 2)$ in $[4,6]$.

In this paper, the stochastic integral of equation (1) is the stochastic pathwise R-S integral. For any $\alpha \in(1-H,(1 / 2))$, the stochastic process $f=\left\{f_{u}, u \in[0, T]\right\}, T<\infty$, belongs to the space $W_{0}^{\alpha, 1}(0, T)$ that is the space of measurable functions $f$ on $[0, T]$ such that

$$
\|f\|_{\alpha, 1}:=\int_{0}^{u} \frac{|f(s)|}{s^{\alpha}} \mathrm{d} s+\int_{0}^{u} \int_{0}^{s} \frac{|f(s)-f(y)|}{(s-y)^{\alpha+1}} \mathrm{~d} y \mathrm{~d} s<\infty,
$$

and then the integral exists (for the details, see [18]). And, the pathwise integral satisfies the following:

$$
\left|\int_{0}^{u} f(s) \mathrm{d} B_{s}^{H}\right| \leq \Lambda\left(\int_{0}^{u} \frac{|f(s)|}{s^{\alpha}} \mathrm{d} s+\int_{0}^{u} \int_{0}^{s} \frac{|f(s)-f(y)|}{(s-y)^{\alpha+1}} \mathrm{~d} y \mathrm{~d} s\right),
$$

where

$$
\Lambda=\frac{1}{\Gamma(1-\alpha)} \sup _{0<s<u<T}\left|\left(D_{u-}^{1-\alpha} B_{u^{-}}\right)(s)\right|
$$

is a random variable. It is well known that $\Lambda$ has finite moments of all orders (see [19]).

2.2. Block Pulse Functions. The definition of BPFs is as follows:

$$
\varphi_{i}(u)= \begin{cases}1, & (i-1) h \leq u<h, i=1,2, \ldots, m, \\ 0, & \text { otherwise }\end{cases}
$$

where $m$ is a positive integer and $h=(1 / m)$ and $u \in[0,1)$.

The BPFs have the following basic properties:

(i) Disjointness:

$$
\varphi_{i}(u) \varphi_{j}(u)=\delta_{i j} \varphi_{i}(u), \quad i, j=1,2, \ldots, m,
$$

where $\delta_{i j}$ is Kronecker delta.

(ii) Orthogonality:

$$
\int_{0}^{1} \varphi_{i}(u) \varphi_{j}(u) \mathrm{d} u=h \delta_{i j}, \quad i, j=1,2, \ldots, m .
$$

(iii) Completeness: if the function $f$ is square integrable, then

$$
\int_{0}^{1} f^{2}(u) \mathrm{d} u=\lim _{m \rightarrow \infty} \sum_{n=1}^{m} f_{n}^{2}\left\|\varphi_{n}(u)\right\|^{2}
$$

where $f_{n}=(1 / h) \int_{0}^{1} f(u) \varphi_{n}(u) \mathrm{d} u$ and the above equation is called Parseval's identity.

The vector form of BPFs is as follows:

$$
\begin{aligned}
\Phi(u) & =\left(\varphi_{1}(u), \varphi_{2}(u), \ldots, \varphi_{m}(u)\right)^{T}, \\
\Phi(u) \Phi^{T}(u) & =\left(\begin{array}{cccc}
\varphi_{1}(u) & 0 & \cdots & 0 \\
0 & \varphi_{2}(u) & \ldots & 0 \\
\vdots & \vdots & \ddots & \vdots \\
0 & 0 & \cdots & \varphi_{m}(u)
\end{array}\right)_{m \times m}, \\
\Phi^{T}(u) \Phi(u) & =1, \\
\Phi(u) \Phi^{T}(u) F & =\mathbf{D}_{F} \Phi(u),
\end{aligned}
$$

where $F=\left(f_{1}, f_{2}, \ldots, f_{m}\right)^{T}$ and $\mathbf{D}_{F}$ denotes a diagonal matrix whose diagonal entries are the constant vector $F$.

2.3. Function Approximation. The square integrable function $x(u)$ on $[0,1)$ can be expressed as

$$
x(u) \simeq x_{m}(u)=\sum_{i=1}^{m} x_{i} \varphi(u)=X^{T} \Phi(u)=\Phi^{T}(u) X,
$$

where 


$$
X=\left(x_{1}, x_{2}, \ldots, x_{m}\right)^{T} .
$$

Let $g(s, u) \in L^{2}([0,1) \times[0,1))$. It can also be approximated as

$$
g(s, u)=\Phi^{T}(s) \mathbf{G} \Psi(u),
$$

where $\Phi(s)$ and $\Psi(s)$ are $m_{1}$ and $m_{2}$ dimensional BPFs vectors, respectively, $\mathbf{G}=\left(g_{i j}\right)_{m_{1} \times m_{2}}$,

$$
g_{i j}=\frac{1}{h_{1} h_{2}} \int_{0}^{1} \int_{0}^{1} g(s, u) \varphi_{i}(s) \psi_{j}(u) \mathrm{d} s \mathrm{~d} u,
$$

and $h_{1}=\left(1 / m_{1}\right)$ and $h_{2}=\left(1 / m_{2}\right)$.

\subsection{Related Lemmas}

Lemma 1 (see [4]). Suppose that $\Phi(u)$ is defined in (10); then, we have

$$
\int_{0}^{u} \Phi(s) \mathrm{d} s \simeq \mathbf{P} \Phi(u)
$$

where

$$
\mathbf{P}=\frac{h}{2}\left(\begin{array}{ccccc}
1 & 2 & 2 & \cdots & 2 \\
0 & 1 & 2 & \cdots & 2 \\
0 & 0 & 1 & \cdots & 2 \\
\vdots & \vdots & \vdots & \ddots & \vdots \\
0 & 0 & 0 & \cdots & 1
\end{array}\right)_{m \times m} .
$$

Lemma 2 (see [12]). The integral of $\Phi(u)$ with respect to FBM has the following approximation:

$$
\int_{0}^{u} \Phi(s) \mathrm{d} B_{s}^{H} \simeq \mathbf{P}_{H} \Phi(u),
$$

where $\mathbf{P}_{H}$ is the stochastic operational matrix with

$$
\mathbf{P}_{H}=\left(\begin{array}{ccclc}
B_{(h / 2)}^{H} & B_{h}^{H} & B_{h}^{H} & \cdots & B_{h}^{H} \\
0 & B_{(3 h / 2)}^{H}-B_{h}^{H} & B_{2 h}^{H}-B_{h}^{H} & \cdots & B_{2 h}^{H}-B_{h}^{H} \\
0 & 0 & B_{(5 h / 2)}^{H}-B_{2 h}^{H} & \cdots & B_{3 h}^{H}-B_{2 h}^{H} \\
\vdots & \vdots & \vdots & \ddots & \vdots \\
0 & 0 & 0 & \cdots & B_{((2 m-1) h / 2)}^{H}-B_{(m-1) h}^{H}
\end{array}\right)_{m \times m}
$$

\section{Numerical Method}

We solve equation (1) by BPFs in this section. To solve this integral equation, a lemma is given to deal with nonlinear analytic functions in the first place.

Lemma 3 (see $[6,7])$. Let $v(u)=\sum a_{j} u^{j}$ and $\kappa(u)=\sum b_{j} u^{j}$ be the analytic functions and $j \in \mathbb{Z}^{+}$; then,

$$
\begin{aligned}
& v\left(x_{m}(u)\right)=v^{T}(X) \Phi(u), \\
& \kappa\left(x_{m}(u)\right)=\kappa^{T}(X) \Phi(u),
\end{aligned}
$$

where

$$
\begin{aligned}
& \nu^{T}(X)=\left(\nu\left(x_{1}\right), v\left(x_{2}\right), \ldots, \nu\left(x_{m}\right)\right), \\
& \kappa^{T}(X)=\left(\kappa\left(x_{1}\right), \kappa\left(x_{2}\right), \ldots, \kappa\left(x_{m}\right)\right) .
\end{aligned}
$$

On the basis of (12) and (14), $x(u), x_{0}(u), g_{1}(s, u)$, and $g_{2}(s, u)$ have the following approximate forms, respectively:

$$
\begin{gathered}
x(u) \simeq X^{T} \Phi(u)=\Phi^{T}(u) X \\
x_{0}(u) \simeq C^{T} \Phi(u)=\Phi^{T}(u) C
\end{gathered}
$$

$$
\begin{aligned}
& g_{1}(s, u) \simeq \Phi^{T}(s) \mathbf{G}_{1} \Psi(u)=\Psi^{T}(u) \mathbf{G}_{1}^{T} \Phi(s), \\
& g_{2}(s, u) \simeq \Phi^{T}(s) \mathbf{G}_{2} \Psi(u)=\Psi^{T}(u) \mathbf{G}_{2}^{T} \Phi(s),
\end{aligned}
$$

where $X$ and $C$ are BPFs coefficients vectors and $\mathbf{G}_{1}$ and $\mathbf{G}_{2}$ are BPFs coefficients matrices similar to (15). For convenience, we put $m_{1}=m_{2}=m$. Substituting above equations into equation (1), we obtain

$$
\begin{aligned}
X^{T} \Phi(u) \simeq & C^{T} \Phi(u)+v^{T}(X) \int_{0}^{u} \Phi(s) \Phi^{T}(s) \mathrm{d} s \mathbf{G}_{1} \Phi(u) \\
& +\kappa^{T}(X) \int_{0}^{u} \Phi(s) \Phi^{T}(s) \mathrm{d} B_{s}^{H} \mathbf{G}_{2} \Phi(u)
\end{aligned}
$$

Applying the operational matrixes $\mathbf{P}$ and $\mathbf{P}_{H}$ for BPFs derived in (16) and (18), equation (23) can be rewritten as

$$
X^{T} \Phi(u) \simeq C^{T} \Phi(u)+v^{T}(X) \mathbf{M}_{1} \Phi(u)+\kappa^{T}(X) \mathbf{M}_{2} \Phi(u),
$$

where $\mathbf{M}_{1}$ and $\mathbf{M}_{2}$ are $m \times m$ matrixes similar to [12]; see appendix, for details, 


$$
\begin{aligned}
& \mathbf{M}_{1}=\frac{h}{2}\left(\begin{array}{ccccc}
g_{11}^{1} & 2 g_{12}^{1} & 2 g_{13}^{1} & \cdots & 2 g_{1 m}^{1} \\
0 & g_{22}^{1} & 2 g_{23}^{1} & \cdots & 2 g_{2 m}^{1} \\
0 & 0 & g_{33}^{1} & \cdots & 2 g_{3 m}^{1} \\
\vdots & \vdots & \vdots & \ddots & \vdots \\
0 & 0 & 0 & \cdots & g_{m m}^{1}
\end{array}\right)_{m \times m} \\
& \mathbf{M}_{2}=\left(\begin{array}{ccccc}
g_{11}^{2} B_{(h / 2)}^{H} & g_{12}^{2} B_{h}^{H} & g_{13}^{2} B_{h}^{H} & \cdots & g_{1 m}^{2} B_{h}^{H} \\
0 & g_{22}^{2}\left(B_{(3 h / 2)}^{H}-B_{h}^{H}\right) & g_{23}^{2}\left(B_{2 h}^{H}-B_{h}^{H}\right) & \cdots & g_{2 m}^{2}\left(B_{2 h}^{H}-B_{h}^{H}\right) \\
0 & 0 & g_{33}^{2}\left(B_{(5 h / 2)}^{H}-B_{2 h}^{H}\right) & \cdots & g_{3 m}^{2}\left(B_{3 h}^{H}-B_{2 h}^{H}\right) \\
\vdots & \vdots & \vdots & \ddots & \vdots \\
0 & 0 & 0 & \cdots & g_{m m}^{2}\left(B_{((2 m-1) h / 2)}^{H}-B_{(m-1) h}^{H}\right)
\end{array}\right)_{m \times m}
\end{aligned}
$$

with $g_{i j}^{1}$ and $g_{i j}^{2}$ are the same as (15).

Then,

$$
X^{T} \simeq C^{T}+v^{T}(X) \mathbf{M}_{1}+\kappa^{T}(X) \mathbf{M}_{2} .
$$

\section{Error Analysis}

The error analysis of the presented method is provided in this section. Firstly, two important lemmas are shown.

Lemma 4 (see [3]). Let $e(s)=f(s)-f_{m}(s)$, where $f_{m}(s)$ is approximations of bounded function $f(s)$ by BPFs on $[0,1)$. Then,

$$
\|e(s)\|_{L^{2}([0,1))}^{2}=\int_{0}^{1} e^{2}(s) \mathrm{d} s \leq O\left(h^{2}\right)
$$

Lemma 5 (see [3]). Let $e(s, u)=g(s, u)-g_{m}(s, u)$, where $g_{m}(s, u)$ is approximations of bounded function $g(s, u)$ by BPFs on $c=[0,1) \times[0,1)$. Then,

$$
\|e(s, u)\|_{L^{2}(c)}^{2}=\int_{0}^{1} \int_{0}^{1} e^{2}(s, u) \mathrm{d} s \mathrm{~d} u \leq O\left(h^{2}\right) .
$$

Secondly, consider $e(u)=x(u)-x_{m}(u)$. We have

$$
\begin{aligned}
e(u)= & x(u)-x_{m}(u) \\
= & x_{0}(u)-x_{0 m}(u) \\
& +\int_{0}^{u}\left[g_{1}(s, u) v(x(s))-g_{1 m}(s, u) v\left(x_{m}(s)\right)\right] \mathrm{d} s \\
& +\int_{0}^{u}\left[g_{2}(s, u) \kappa(x(s))-g_{2 m}(s, u) \kappa\left(x_{m}(s)\right)\right] \mathrm{d} B_{s}^{H},
\end{aligned}
$$

where $x_{m}(u), x_{0 m}(u), g_{1 m}(s, u)$, and $g_{2 m}(s, u)$ are approximation of $x(u), x_{0}(u), g_{1}(s, u)$, and $g_{2}(s, u)$ by BPFs, respectively.

Then, for each $R>1$, we define the following stopping time $\tau_{R}$ :

$$
\tau_{R}:=\inf \{u \geq 0: \Lambda \geq R\} \wedge T .
$$

Finally, we give the main theorem and prove it.

Theorem 1. Assume that bounded analytic functions $\nu(x(u))$ and $\kappa(x(u))$ satisfy Lipschitz conditions:

$$
\begin{aligned}
& \text { (I) }|v(q)-v(p)| \leq l_{1}|q-p| \text { and }|\kappa(q)-\kappa(p)| \\
& \quad \leq l_{3}|q-p| \\
& \text { (II) }|v(q)| \leq l_{2} \text { and }|\kappa(q)| \leq l_{4} \\
& \text { (III) }\left|g_{1}(s, u)\right| \leq l_{5} \text { and }\left|g_{2}(s, u)\right| \leq l_{6} \\
& \text { In (I)-(III), } q, p \in \mathbb{R}, l_{i}>0, i=1, \ldots, 6 \text { are constants. }
\end{aligned}
$$
Then,

$$
\begin{array}{rl}
\int_{0}^{T} & \mathbb{E}\left(\left|e\left(u \wedge \tau_{R}\right)\right|^{2}\right) \mathrm{d} u \\
\quad=\int_{0}^{T} \mathbb{E}\left(\left|x\left(u \wedge \tau_{R}\right)-x_{m}\left(u \wedge \tau_{R}\right)\right|^{2}\right) \mathrm{d} u \\
\quad \leq C(R) O\left(h^{2-4 \alpha}\right), \quad T \in[0,1),
\end{array}
$$




$$
\begin{aligned}
& \mathbb{E}\left(\left|e\left(u \wedge \tau_{R}\right)\right|^{2}\right) \leq 3\left[\mathbb{E}\left(\left|x_{0}(u)-x_{0 m}(u)\right|^{2}\right)\right. \\
& \quad+\mathbb{E}\left(\left|\int_{0}^{u \wedge \tau_{R}}\left[g_{1}\left(s, u \wedge \tau_{R}\right) v(x(s))-g_{1 m}\left(s, u \wedge \tau_{R}\right) v\left(x_{m}(s)\right)\right] \mathrm{d} s\right|^{2}\right) \\
& \left.\quad+\mathbb{E}\left(\left|\int_{0}^{u \wedge \tau_{R}}\left[g_{2}\left(s, u \wedge \tau_{R}\right) \kappa(x(s))-g_{2 m}\left(s, u \wedge \tau_{R}\right) \kappa\left(x_{m}(s)\right)\right] \mathrm{d} B_{s}^{H}\right|^{2}\right)\right]:=3 J_{1}+3 J_{2}+3 J_{3} .
\end{aligned}
$$

For the second item, using Lipschitz conditions and Cauchy-Schwarz inequality, we have

$$
\begin{aligned}
J_{2} \leq & \int_{0}^{u} \mathbb{E}\left(\mid g_{1}\left(s, u \wedge \tau_{R}\right)\left[v(x(s))-v\left(x_{m}(s)\right)\right]\right. \\
& \left.+\left.v\left(x_{m}(s)\right)\left[g_{1}\left(s, u \wedge \tau_{R}\right)-g_{1 m}\left(s, u \wedge \tau_{R}\right)\right]\right|^{2}\right) \mathrm{d} s \\
\leq & 2 l_{1}^{2} l_{5}^{2} \int_{0}^{u} \mathbb{E}\left(|e(s)|^{2}\right) \mathrm{d} s \\
& +2 l_{2}^{2} \int_{0}^{u}\left|g_{1}\left(s, u \wedge \tau_{R}\right)-g_{1 m}\left(s, u \wedge \tau_{R}\right)\right|^{2} \mathrm{~d} s .
\end{aligned}
$$

$$
\begin{aligned}
J_{3} \leq & 2 \mathbb{E}\left(\Lambda \int_{0}^{u} \mid g_{2}\left(s, u \wedge \tau_{R}\right) \kappa(x(s))\right. \\
& \left.-g_{2 m}\left(s, u \wedge \tau_{R}\right) \kappa\left(x_{m}(s)\right) \mid s^{-\alpha} \mathrm{d} s\right)^{2} \\
& +2 \mathbb{E}\left(\Lambda \int_{0}^{u} \int_{0}^{s} \mid g_{2}\left(s, u \wedge \tau_{R}\right) \kappa(x(s))\right. \\
& -g_{2 m}(x(s)) \kappa\left(x_{m}(s)\right)-g_{2}\left(y, u \wedge \tau_{R}\right) \kappa(x(y)) \\
& \left.+g_{2 m}\left(y, u \wedge \tau_{R}\right) \kappa\left(x_{m}(y)\right) \mid(s-y)^{-\alpha-1} \mathrm{~d} y \mathrm{~d} s\right)^{2}:=J_{31}+J_{32} .
\end{aligned}
$$

Then,

For the last item, by (4), we obtain

$$
\begin{aligned}
J_{31} \leq & 2 R^{2} \mathbb{E}\left(\int_{0}^{u} \mid g_{2}\left(s, u \wedge \tau_{R}\right)\left[\kappa(x(s))-\kappa\left(x_{m}(s)\right)\right]\right. \\
& \left.+\kappa\left(x_{m}(s)\right)\left[g_{2}\left(s, u \wedge \tau_{R}\right)-g_{2 m}\left(s, u \wedge \tau_{R}\right)\right] \mid s^{-\alpha} \mathrm{d} s\right)^{2} \\
\leq & 4 R^{2} l_{3}^{2} l_{6}^{2} \int_{0}^{u} \mathbb{E}\left(|e(s)|^{2}\right) \mathrm{d} s+4 R^{2} l_{4}^{2} \\
& \left.\times \int_{0}^{u}\left|g_{2}\left(s, u \wedge \tau_{R}\right)-g_{2 m}\left(s, u \wedge \tau_{R}\right)\right|^{2} \mathrm{~d} s\right] \int_{0}^{u} s^{-2 \alpha} \mathrm{d} s \\
\leq & 4 R^{2} l_{3}^{2} l_{6}^{2} C_{0} \int_{0}^{u} \mathbb{E}\left(|e(s)|^{2}\right) \mathrm{d} s \\
& +4 R^{2} l_{4}^{2} C_{0} \int_{0}^{u}\left|g_{2}\left(s, u \wedge \tau_{R}\right)-g_{2 m}\left(s, u \wedge \tau_{R}\right)\right|^{2} \mathrm{~d} s, \\
J_{32} \leq & 2 R^{2} \mathbb{E}\left(\int_{0}^{u} \int_{0}^{s} \mid g_{2}\left(s, u \wedge \tau_{R}\right)\left[\kappa(x(s))-\kappa\left(x_{m}(s)\right)\right]+\mu\left(x_{m}(s)\right)\left[g_{2}\left(s, u \wedge \tau_{R}\right)-g_{2 m}\left(s, u \wedge \tau_{R}\right)\right]\right. \\
& \left.-g_{2}\left(y, u \wedge \tau_{R}\right)\left[\kappa(x(y))-\kappa\left(x_{m}(y)\right)\right]-\mu\left(x_{m}(y)\right)\left[g_{2}\left(y, u \wedge \tau_{R}\right)-g_{2 m}\left(y, u \wedge \tau_{R}\right)\right] \mid(s-y)^{-\alpha-1} \mathrm{~d} y \mathrm{~d} s\right)^{2} \\
\leq & 8 R^{2} l_{3}^{2} l_{6}^{2} \mathbb{E}\left(\int_{0}^{u} \int_{0}^{u_{s}}|e(s)|(s-y)^{-\alpha-1} \mathrm{~d} y \mathrm{~d} s+\int_{0}^{u} \int_{u_{s}}^{s}|e(s)|(s-y)^{-\alpha-1} \mathrm{~d} y \mathrm{~d} s\right)^{2} \\
& +8 R^{2} l_{4}^{2}\left(\int_{0}^{u} \int_{0}^{u_{s}}\left|g_{2}\left(s, u \wedge \tau_{R}\right)-g_{2 m}\left(s, u \wedge \tau_{R}\right)\right|(s-y)^{-\alpha-1} \mathrm{~d} y \mathrm{~d} s\right. \\
& \left.+\int_{0}^{u} \int_{u_{s}}^{s}\left|g_{2}\left(s, u \wedge \tau_{R}\right)-g_{2 m}\left(s, u \wedge \tau_{R}\right)\right|(s-y)^{-\alpha-1} \mathrm{~d} y \mathrm{~d} s\right)^{2} \\
& +8 R^{2} l_{3}^{2} l_{6}^{2} \mathbb{E}\left(\int_{0}^{u} \int_{u_{y}}^{u}|e(y)|(s-y)^{-\alpha-1} \mathrm{~d} y \mathrm{~d} s+\int_{0}^{u} \int_{y}^{u_{y}}|e(y)|(s-y)^{-\alpha-1} \mathrm{~d} y \mathrm{~d} s\right)^{2} \\
& +8 R^{2} l_{4}^{2}\left(\int_{0}^{u} \int_{u_{y}}^{u}\left|g_{2}\left(y, u \wedge \tau_{R}\right)-g_{2 m}\left(y, u \wedge \tau_{R}\right)\right|(s-y)^{-\alpha-1} \mathrm{~d} y \mathrm{~d} s\right. \\
& \left.+\int_{0}^{u} \int_{y}^{u_{y}}\left|g_{2}\left(y, u \wedge \tau_{R}\right)-g_{2 m}\left(y, u \wedge \tau_{R}\right)\right|(s-y)^{-\alpha-1} \mathrm{~d} y \mathrm{~d} s\right)^{2}
\end{aligned}
$$




$$
\begin{aligned}
\leq & 8 R^{2} l_{3}^{2} l_{6}^{2}\left(\alpha^{-1}+1\right)^{2} \int_{0}^{u} \mathbb{E}\left(|e(s)|^{2}\right) \mathrm{d} s \int_{0}^{u}\left(s-u_{s}\right)^{-2 \alpha} \mathrm{d} s \\
& +8 R^{2} l_{4}^{2}\left(\alpha^{-1}+1\right)^{2} \int_{0}^{u}\left|g_{2}\left(s, u \wedge \tau_{R}\right)-g_{2 m}\left(s, u \wedge \tau_{R}\right)\right|^{2} \mathrm{~d} s \int_{0}^{u}\left(s-u_{s}\right)^{-2 \alpha} \mathrm{d} s \\
& +8 R^{2} l_{3}^{2} l_{6}^{2}\left(\alpha^{-1}+1\right)^{2} \mathbb{E}\left(\int_{0}^{u}|e(y)|\left[\left(u_{y}-y\right)^{-\alpha}-(u-y)^{-\alpha}\right] \mathrm{d} y\right)^{2} \\
& +8 R^{2} l_{4}^{2}\left(\alpha^{-1}+1\right)^{2}\left(\int_{0}^{u}\left|g_{2}\left(y, u \wedge \tau_{R}\right)-g_{2 m}\left(y, u \wedge \tau_{R}\right)\right|\left[\left(u_{y}-y\right)^{-\alpha}-(u-y)^{-\alpha}\right] \mathrm{d} y\right)^{2} \\
\leq & 8 R^{2} l_{3}^{2} l_{6}^{2} C_{1} h^{-2 \alpha} \int_{0}^{u} \mathbb{E}\left(|e(s)|^{2}\right) \mathrm{d} s+8 R^{2} l_{4}^{2} C_{1} h^{-2 \alpha} \int_{0}^{u}\left|g_{2}\left(s, u \wedge \tau_{R}\right)-g_{2 m}\left(s, u \wedge \tau_{R}\right)\right|^{2} \mathrm{~d} s \\
& +8 R^{2} l_{3}^{2} l_{6}^{2} C_{1} h^{-2 \alpha} \int_{0}^{u} \mathbb{E}\left(|e(y)|^{2}\right) \mathrm{d} y+8 R^{2} l_{4}^{2} C_{1} h^{-2 \alpha} \int_{0}^{u}\left|g_{2}\left(y, u \wedge \tau_{R}\right)-g_{2 m}\left(y, u \wedge \tau_{R}\right)\right|^{2} \mathrm{~d} y,
\end{aligned}
$$

where $\varsigma_{n}=(n / m)=n h, n=0, \ldots, m, u_{s}=\max \left\{\varsigma_{n}: \varsigma_{n} \leq s\right\}$, $u_{y}=\min \left\{\varsigma_{n}: \varsigma_{n} \geq y\right\}$, and $C_{0}=(1-2 \alpha)^{-1}$ and

Finally, by combining (33)-(36), we obtain $C_{1}=C_{0}\left(\alpha^{-1}+1\right)^{2}$.

$$
\begin{aligned}
\mathbb{E}\left(\left|e\left(u \wedge \tau_{R}\right)\right|^{2}\right) \leq & 3\left[\left|x_{0}(u)-x_{0 m}(u)\right|^{2}\right. \\
& +2 l_{1}^{2} l_{5}^{2} \int_{0}^{u} \mathbb{E}\left(|e(s)|^{2}\right) \mathrm{d} s+2 l_{2}^{2} \int_{0}^{u}\left|g_{1}\left(s, u \wedge \tau_{R}\right)-g_{1 m}\left(s, u \wedge \tau_{R}\right)\right|^{2} \mathrm{~d} s \\
& +4 R^{2} l_{3}^{2} l_{6}^{2} C_{0} \int_{0}^{u} \mathbb{E}\left(|e(s)|^{2}\right) \mathrm{d} s+4 R^{2} l_{4}^{2} C_{0} \times \int_{0}^{u}\left|g_{2}\left(s, u \wedge \tau_{R}\right)-g_{2 m}\left(s, u \wedge \tau_{R}\right)\right|^{2} \mathrm{~d} s \\
& +8 R^{2} l_{3}^{2} l_{6}^{2} C_{1} h^{-2 \alpha} \int_{0}^{u} \mathbb{E}\left(|e(s)|^{2}\right) \mathrm{d} s+8 R^{2} l_{4}^{2} C_{1} h^{-2 \alpha} \\
& \times \int_{0}^{u}\left|g_{2}\left(s, u \wedge \tau_{R}\right)-g_{2 m}\left(s, u \wedge \tau_{R}\right)\right|^{2} \mathrm{~d} s+8 R^{2} l_{3}^{2} l_{6}^{2} C_{1} h^{-2 \alpha} \int_{0}^{u} \mathbb{E}\left(|e(y)|^{2}\right) \mathrm{d} y \\
& \left.+8 R^{2} l_{4}^{2} C_{1} h^{-2 \alpha} \times \int_{0}^{u}\left|g_{2}\left(y, u \wedge \tau_{R}\right)-g_{2 m}\left(y, u \wedge \tau_{R}\right)\right|^{2} \mathrm{~d} y\right] .
\end{aligned}
$$

Then,

$$
\begin{aligned}
\mathbb{E}\left(\left|e\left(u \wedge \tau_{R}\right)\right|^{2}\right) \leq & 3\left[\left|x_{0}(u)-x_{0 m}(u)\right|^{2}\right. \\
& +2 l_{2}^{2} \int_{0}^{u}\left|g_{1}\left(s, u \wedge \tau_{R}\right)-g_{1 m}\left(s, u \wedge \tau_{R}\right)\right|^{2} \mathrm{~d} s+\left(4 R^{2} l_{4}^{2} C_{0}+16 R^{2} l_{4}^{2} C_{1} h^{-2 \alpha}\right) \\
& \left.\times \int_{0}^{u}\left|g_{2}\left(s, u \wedge \tau_{R}\right)-g_{2 m}\left(s, u \wedge \tau_{R}\right)\right|^{2} \mathrm{~d} s\right]+\left(6 l_{1}^{2} l_{5}^{2}+12 R^{2} l_{3}^{2} l_{6}^{2} C_{0}+48 R^{2} l_{3}^{2} l_{6}^{2} C_{1} h^{-2 \alpha}\right) \\
& \times \int_{0}^{u} \mathbb{E}\left(|e(s)|^{2}\right) \mathrm{d} s,
\end{aligned}
$$

or

$$
\mathbb{E}\left(\left|e\left(u \wedge \tau_{R}\right)\right|^{2}\right) \leq \sigma(u)+\lambda \int_{0}^{u} \mathbb{E}\left(|e(s)|^{2}\right) \mathrm{d} s,
$$


TABLE 1: When $m=16$ and $H=0.6$, the table exhibits numerical results of Example 1 .

\begin{tabular}{cccrr}
\hline$u$ & \multirow{2}{*}{$X_{E}$} & $X_{S}$ & \multicolumn{2}{c}{$95 \%$ confidence interval for error mean } \\
& & & Lower & Upper \\
\hline $1 / 16$ & $1.23683212 E-05$ & $6.18416058 E-06$ & $2.47366423 E-07$ & $2.44892759 E-05$ \\
$2 / 16$ & $2.15162737 E-05$ & $1.07581368 E-05$ & $4.30325473 E-07$ & $4.26022219 E-05$ \\
$3 / 16$ & $2.89145059 E-05$ & $1.44572530 E-05$ & $5.78290119 E-07$ & $5.72507217 E-05$ \\
$4 / 16$ & $3.48716777 E-05$ & $1.74358389 E-05$ & $6.97433554 E-07$ & $6.90459219 E-05$ \\
$5 / 16$ & $4.00955361 E-05$ & $2.00477681 E-05$ & $8.01910722 E-07$ & $7.93891615 E-05$ \\
\hline
\end{tabular}

TABLE 2: When $m=16$ and $H=0.8$, the table exhibits numerical results of Example 1.

\begin{tabular}{cccrr}
\hline$u$ & $X_{E}$ & $X_{S}$ & \multicolumn{2}{c}{$95 \%$ confidence interval for error mean } \\
& & & Lower & Upper \\
\hline $1 / 16$ & $3.87689012 E-06$ & $1.93844506 E-06$ & $7.75378025 E-08$ & $7.67624244 E-06$ \\
$2 / 16$ & $8.79318940 E-06$ & $4.39659470 E-06$ & $1.75863788 E-07$ & $1.74105150 E-05$ \\
$3 / 16$ & $1.29658037 E-05$ & $6.48290186 E-06$ & $2.59316074 E-07$ & $2.56722914 E-05$ \\
$4 / 16$ & $1.86048116 E-05$ & $9.30240580 E-06$ & $3.72096232 E-07$ & $3.68375270 E-05$ \\
$5 / 16$ & $2.14168523 E-05$ & $1.07084262 E-05$ & $4.28337047 E-07$ & $4.24053677 E-05$ \\
\hline
\end{tabular}

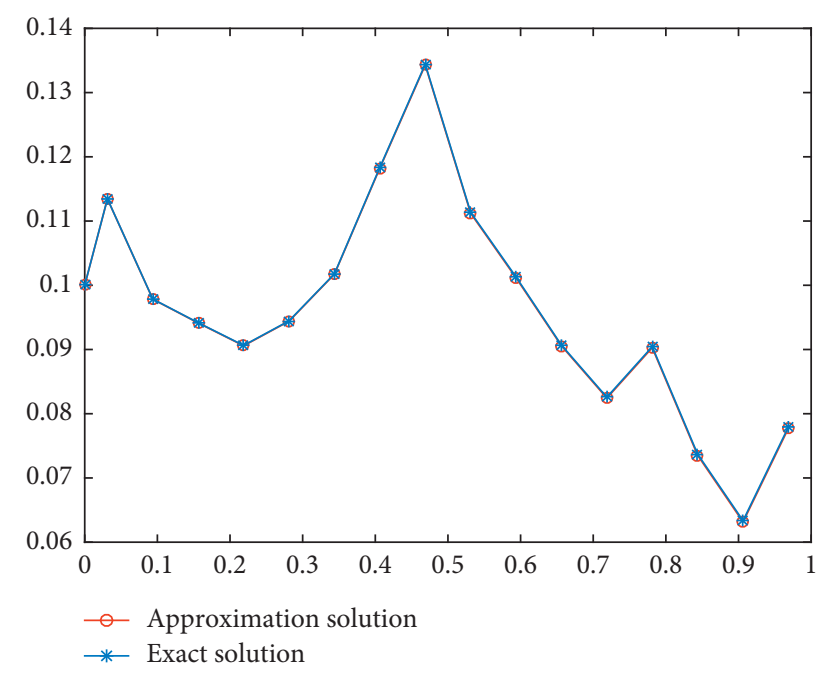

FIgURE 1: Simulation result of Example 1 for $H=0.6$ and $m=16$.

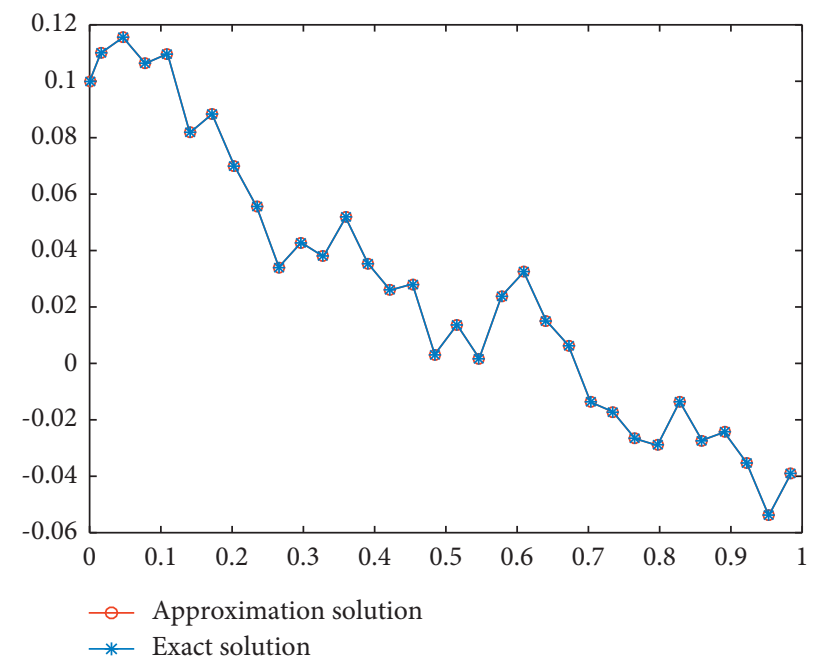

FIgURE 2: Simulation result of Example 1 for $H=0.6$ and $m=32$. 
TABLE 3: When $m=16$ and $H=0.6$, the table exhibits numerical results of Example 2.

\begin{tabular}{cccrr}
\hline$u$ & \multirow{2}{*}{$X_{E}$} & $X_{S}$ & \multicolumn{2}{c}{$95 \%$ confidence interval for error mean } \\
& & & Lower & Upper \\
\hline $1 / 16$ & $4.82207134 E-06$ & $2.41103567 E-06$ & $9.64414269 E-08$ & $9.54770126 E-05$ \\
$2 / 16$ & $9.11303123 E-06$ & $4.55651561 E-06$ & $1.82260625 E-07$ & $1.80438018 E-05$ \\
$3 / 16$ & $1.31576729 E-05$ & $6.57883646 E-06$ & $2.63153458 E-07$ & $2.60521924 E-05$ \\
$4 / 16$ & $1.60910855 E-05$ & $8.04554277 E-06$ & $3.21821711 E-07$ & $3.18603494 E-05$ \\
$5 / 16$ & $2.03276041 E-05$ & $1.01638020 E-05$ & $4.06552081 E-07$ & $4.02486560 E-05$ \\
\hline
\end{tabular}

TABLE 4: When $m=16$ and $H=0.8$, the table exhibits numerical results of Example 2.

\begin{tabular}{cccrr}
\hline$u$ & \multirow{2}{*}{$X_{E}$} & $X_{S}$ & \multicolumn{2}{c}{$95 \%$ confidence interval for error mean } \\
& & & Lower & Upper \\
\hline $1 / 16$ & $3.08317271 E-06$ & $1.54158636 E-06$ & $6.16634543 E-08$ & $6.10468197 E-06$ \\
$2 / 16$ & $6.35447091 E-06$ & $3.17723546 E-06$ & $1.27089418 E-07$ & $1.25818524 E-05$ \\
$3 / 16$ & $7.04978090 E-06$ & $3.52489045 E-06$ & $1.40995618 E-07$ & $1.39585662 E-05$ \\
$4 / 16$ & $8.48079420 E-06$ & $4.24039710 E-06$ & $1.69615884 E-07$ & $1.67919725 E-05$ \\
$5 / 16$ & $1.14691035 E-05$ & $5.73455173 E-06$ & $2.29382069 E-07$ & $2.27088249 E-05$ \\
\hline
\end{tabular}

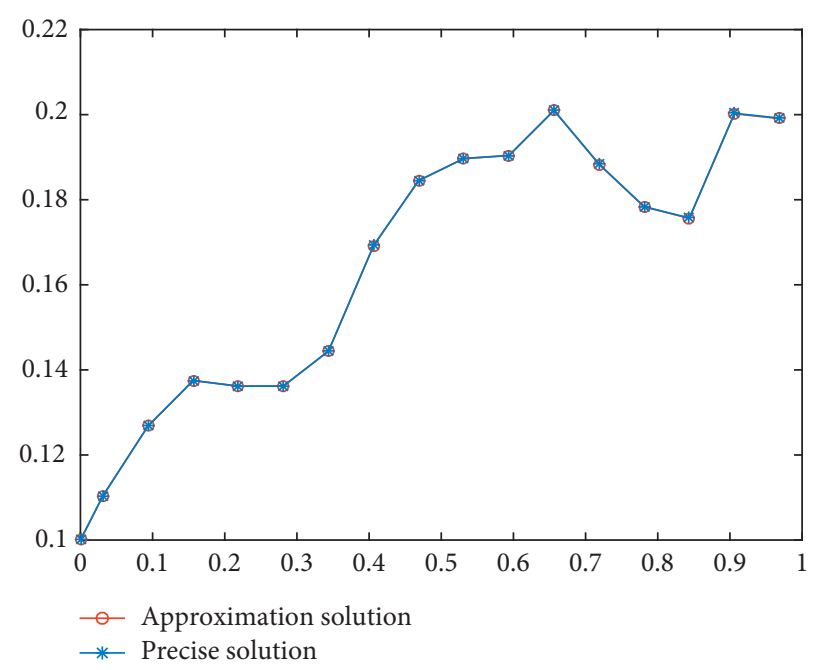

Figure 3: Simulation result of Example 2 for $H=0.8$ and $m=16$.

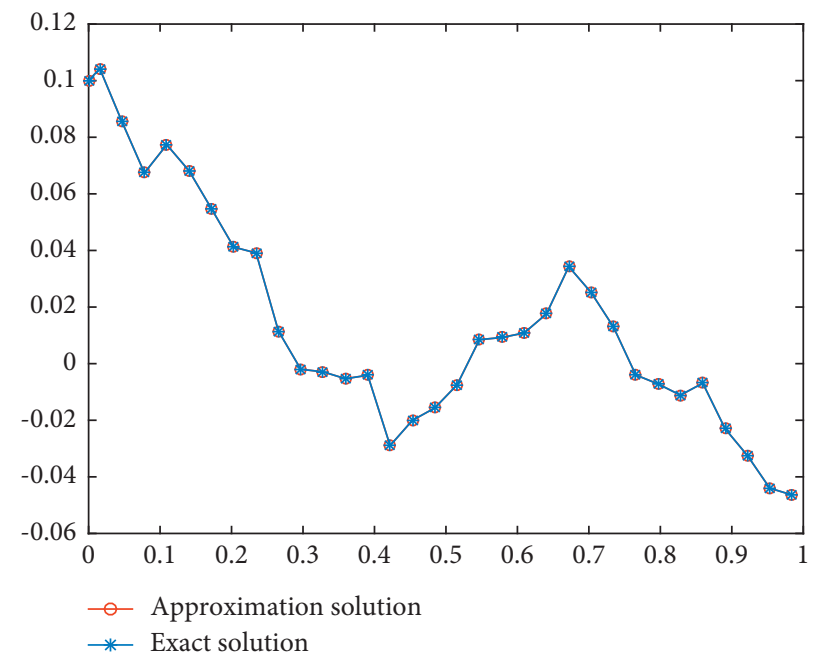

Figure 4: Simulation result of Example 2 for $H=0.8$ and $m=32$. 


$$
\begin{aligned}
\sigma(u)= & 3\left[\left|x_{0}(u)-x_{0 m}(u)\right|^{2}+2 l_{2}^{2} \int_{0}^{u}\left|g_{1}\left(s, u \wedge \tau_{R}\right)-g_{1 m}\left(s, u \wedge \tau_{R}\right)\right|^{2} \mathrm{~d} s\right. \\
& +\left(4 R^{2} l_{4}^{2} C_{0}+16 R^{2} l_{4}^{2} C_{1} h^{-2 \alpha}\right) \\
& \left.\times \int_{0}^{u}\left|g_{2}\left(s, u \wedge \tau_{R}\right)-g_{2 m}\left(s, u \wedge \tau_{R}\right)\right|^{2} \mathrm{~d} s\right] \\
\lambda= & 6 l_{1}^{2} l_{5}^{2}+12 R^{2} l_{3}^{2} l_{6}^{2} C_{0}+48 R^{2} l_{3}^{2} l_{6}^{2} C_{1} h^{-2 \alpha} .
\end{aligned}
$$

Let $V\left(u \wedge \tau_{R}\right)=\mathbb{E}\left(\left|e\left(u \wedge \tau_{R}\right)\right|^{2}\right)$; we obtain $V\left(u \wedge \tau_{R}\right) \leq \sigma(u)+\lambda \int_{0}^{u} e^{\lambda\left(u \wedge \tau_{R}-\theta\right)} \sigma(\theta) \mathrm{d} \theta, \quad u \in[0, T)$.

$$
V\left(u \wedge \tau_{R}\right) \leq \sigma(u)+\lambda \int_{0}^{u} V(\theta) \mathrm{d} \theta, \quad \theta \in[0, u) .
$$

According to Gronwall's inequality, we have

Then,

$$
\begin{aligned}
\int_{0}^{T} V\left(u \wedge \tau_{R}\right) \mathrm{d} u= & \int_{0}^{T} \mathbb{E}\left(\left|e\left(u \wedge \tau_{R}\right)\right|^{2}\right) \mathrm{d} u \\
\leq & \int_{0}^{T}\left(\sigma(u)+\lambda \int_{0}^{u} e^{\lambda\left(u \wedge \tau_{R}-\theta\right)} \sigma(\theta) \mathrm{d} \theta\right) \mathrm{d} u \\
= & \int_{0}^{T} \sigma(u) \mathrm{d} u+\lambda \int_{0}^{T} \int_{0}^{u} e^{\lambda\left(u \wedge \tau_{R}-\theta\right)} \sigma(\theta) \mathrm{d} \theta \mathrm{d} u \\
\leq & \int_{0}^{T} \sigma(u) \mathrm{d} u+\lambda e^{\lambda T} \int_{0}^{T} \int_{0}^{u} \sigma(\theta) \mathrm{d} \theta \mathrm{d} u=3 \int_{0}^{T}\left|x_{0}(u)-x_{0 m}(u)\right|^{2} \mathrm{~d} u \\
& +6 l_{2}^{2} \int_{0}^{T} \int_{0}^{u}\left|g_{1}\left(s, u \wedge \tau_{R}\right)-g_{1 m}\left(s, u \wedge \tau_{R}\right)\right|^{2} \mathrm{~d} s \mathrm{~d} u \\
& +\left(12 R^{2} l_{4}^{2} C_{0}+48 R^{2} l_{4}^{2} C_{1} h^{-2 \alpha}\right) \int_{0}^{T} \int_{0}^{u}\left|g_{2}\left(s, u \wedge \tau_{R}\right)-g_{2 m}\left(s, u \wedge \tau_{R}\right)\right|^{2} \mathrm{~d} s \mathrm{~d} u \\
& +\lambda e^{\lambda T}\left[3 \int_{0}^{T} \int_{0}^{u}\left|x_{0}(\theta)-x_{0 m}(\theta)\right|^{2} \mathrm{~d} \theta \mathrm{d} u\right. \\
& +6 l_{2}^{2} \int_{0}^{T} \int_{0}^{u} \int_{0}^{\theta}\left|g_{1}\left(s, \theta \wedge \tau_{R}\right)-g_{1 m}\left(s, \theta \wedge \tau_{R}\right)\right|^{2} \mathrm{~d} s \mathrm{~d} \theta \mathrm{d} u \\
& \left.+\left(12 R^{2} l_{4}^{2} C_{0}+48 R^{2} l_{4}^{2} C_{1} h^{-2 \alpha}\right) \int_{0}^{T} \int_{0}^{u} \int_{0}^{\theta}\left|g_{2}\left(s, \theta \wedge \tau_{R}\right)-g_{2 m}\left(s, \theta \wedge \tau_{R}\right)\right|^{2} \mathrm{~d} s \mathrm{~d} \theta \mathrm{d} u\right] \\
:= & 3 N_{1}+6 l_{2}^{2} N_{2}+\left(12 R^{2} l_{4}^{2} C_{0}+48 R^{2} l_{4}^{2} C_{1} h^{-2 \alpha}\right) N_{3} \\
& +\lambda e^{\lambda T}\left[3 N_{4}+6 l_{2}^{2} N_{5}+\left(12 R^{2} l_{4}^{2} C_{0}+48 R^{2} l_{4}^{2} C_{1} h^{-2 \alpha}\right) N_{6}\right] .
\end{aligned}
$$

By (27) and (28), we have

$$
N_{i} \leq w_{i} h^{2}, \quad i=1, \ldots, 6 .
$$

So,

$$
\begin{aligned}
& \int_{0}^{T} \mathbb{E}\left(\left|e\left(u \wedge \tau_{R}\right)\right|^{2}\right) \mathrm{d} u \leq\left[3 w_{1}+6 l_{2}^{2} w_{2}\right. \\
& \quad+\left(12 R^{2} l_{4}^{2} C_{0}+48 R^{2} l_{4}^{2} C_{1} h^{-2 \alpha}\right) w_{3} \\
& \quad+\lambda e^{\lambda T}\left(3 w_{4}+6 l_{2}^{2} w_{5}+12 R^{2} l_{4}^{2} C_{0} w_{6}\right. \\
& \left.\left.\quad+48 R^{2} l_{4}^{2} C_{1} h^{-2 \alpha} w_{6}\right)\right] h^{2} \leq C(R) O\left(h^{2-4 \alpha}\right),
\end{aligned}
$$

where $w_{i}>0, i=1, \ldots, 6$, are constants.

The proof is accomplished.

\section{Numerical Example}

We verify the effectiveness and precision of the above method by two numerical examples in this section.

Example 1. The following nonlinear SIVIE is discussed [8]:

$$
\begin{aligned}
x(u)= & x_{0}-2 H \beta^{2} \int_{0}^{u} s^{2 H-1} x(s)\left(1-x^{2}(s)\right) \mathrm{d} s \\
& +\beta \int_{0}^{u}\left(1-x^{2}(s)\right) \mathrm{d} B_{s}^{H}, \quad u \in[0,1),
\end{aligned}
$$

where $x(u)=\tanh \left(\beta B_{u}^{H}+\operatorname{arctanh}\left(x_{0}\right)\right)$ is the exact solution. 
Let $\beta=(1 / 30)$ and $x_{0}=(1 / 10)$. For different values of $H$, the error means $X_{E}$, error standard deviations $X_{S}$, and confidence intervals for error means of Example 1 with $m=$ 16 are given in Tables 1 and 2 , where $X_{E}$ and $X_{S}$ are obtained by $10^{2}$ trajectories. Also, when $m=16$ and $H=0.6$, the simulation result of the approximate and precise solutions of this example are shown in Figure 1, and for $m=32$ and $H=0.6$, the results are exhibited in Figure 2.

Example 2. The following nonlinear SIVIE is discussed [8]:

$$
\begin{aligned}
x(u)= & x_{0}-H \beta^{2} \int_{0}^{u} \tanh (x(s)) s^{2 H-1} \operatorname{sech}^{2}(x(s)) \mathrm{d} s \\
& +\beta \int_{0}^{t} \operatorname{sech}(x(s)) \mathrm{d} B_{s}^{H}, \quad u \in[0,1),
\end{aligned}
$$

where $x(u)=\operatorname{arcsinh}\left(\beta B_{u}^{H}+\sinh \left(x_{0}\right)\right)$ is the exact solution.

Let $\beta=(1 / 30)$ and $x_{0}=(1 / 10)$. Similarly, for different values of $H, X_{E}$, and $X_{S}$ and confidence intervals for error means of Example 2 with $m=16$ are given in Tables 3 and 4 , where $X_{E}$ and $X_{S}$ are obtained by $10^{2}$ trajectories. Also, when $m=16$ and $H=0.8$, simulation result of the approximate and precise solutions of this example is shown in Figure 3, and for $m=32$ and $H=0.8$, the results are shown in Figure 4.

\section{Conclusion}

For nonlinear SIVIEs driven by FBM, this paper presents a valid method to solve them by BPFs. The simulation results show that the approximate solution of this method is very close to the exact solution. In Section 4, error analysis is given, and the convergence rate of the approximate solution is faster than the method of [21] when $H \longrightarrow 1$.

\section{Appendix}

In this section, we give detailed proofs of $\mathbf{M}_{1}$ and $\mathbf{M}_{2}$.

Let $G_{1}^{i}$ and $G_{2}^{i}$ be the $i$ th row of the matrix $\mathbf{G}_{1}$ and $\mathbf{G}_{2}$, respectively, $R^{i}$ and $R_{H}^{i}$ be the $i$ th row of the integral operation matrix $\mathbf{P}$ and the stochastic integral matrix $\mathbf{P}_{H}$, respectively, and $\mathbf{D}_{G_{1}^{i}}$ and $\mathbf{D}_{G_{2}^{i}}$ be a diagonal matrix with $G_{1}^{i}$ and $G_{2}^{i}$ as the diagonal elements, respectively. By (16) and (18), we obtain

$$
\begin{aligned}
\left(\int_{0}^{u} \Phi(s) \Phi(s)^{T} \mathrm{~d} s\right) \mathbf{G}_{1} \Phi(u) & =\left(\begin{array}{c}
R^{1} \Phi(u) G_{1}^{1} \Phi(u) \\
R^{2} \Phi(u) G_{1}^{2} \Phi(u) \\
\vdots \\
R^{m} \Phi(u) G_{1}^{m} \Phi(u)
\end{array}\right) \\
& =\left(\begin{array}{c}
R^{1} \mathbf{D}_{G_{1}^{1}} \\
R^{2} \mathbf{D}_{G_{1}^{2}} \\
\vdots \\
R^{m} \mathbf{D}_{G_{1}^{m}}
\end{array}\right) \Phi(u)=\mathbf{M}_{1} \Phi(u),
\end{aligned}
$$

$$
\begin{aligned}
\left(\int_{0}^{u} \Phi(s) \Phi(s)^{T} \mathrm{~d} B_{s}^{H}\right) \mathbf{G}_{2} \Phi(u) & =\left(\begin{array}{c}
R_{H}^{1} \Phi(u) G_{2}^{1} \Phi(u) \\
R_{H}^{2} \Phi(u) G_{2}^{2} \Phi(u) \\
\vdots \\
R_{H}^{m} \Phi(u) G_{2}^{m} \Phi(u)
\end{array}\right) \\
& =\left(\begin{array}{c}
R_{H}^{1} \mathbf{D}_{G_{2}^{1}} \\
R_{H}^{2} \mathbf{D}_{G_{2}^{2}} \\
\vdots \\
R_{H}^{m} \mathbf{D}_{G_{2}^{m}}
\end{array}\right) \Phi(u)=\mathbf{M}_{2} \Phi(u) .
\end{aligned}
$$

\section{Data Availability}

No data were used to support this study.

\section{Conflicts of Interest}

The authors state that there are no conflicts of interest in the publication of this paper.

\section{Acknowledgments}

This article was funded by the NSF (Grant 2016CFB526) of Hubei Province.

\section{References}

[1] M. Asgari, E. Hashemizadeh, M. Khodabin, and K. Maleknejad, "Numerical solution of nonlinear stochastic integral equation by stochastic operational matrix based on Bernstein polynomials," Bulletin mathématique de la Société des Sciences Mathématiques de Roumanie, vol. 57, no. 1, pp. 3-12, 2014.

[2] W. F. Blyth, R. L. May, and P. Widyaningsih, "Volterra integral equations solved in Fredholm form using Walsh functions," Anziam Journal, vol. 45, pp. 269-282, 2003.

[3] K. Maleknejad, M. Khodabin, and M. Rostami, "A numerical method for solving $\mathrm{m}$-dimensional stochastic Itô-Volterra integral equations by stochastic operational matrix," Computers \& Mathematics with Applications, vol. 63, no. 1, pp. 133-143, 2012.

[4] K. Maleknejad, M. Khodabin, and M. Rostami, "Numerical solution of stochastic Volterra integral equation by a stochastic operational matrix based on block pulse function," Mathematical and Computer Modelling, vol. 55, no. 3-4, pp. 791-800, 2012.

[5] F. Mohammadi, "Numerical solution of stochastic Itô-Voltterra integral equations using Haar wavelets," Numerical Mathematics: Theory, Methods and Applications, vol. 9, no. 3, pp. 416-431, 2016.

[6] X. Y. Sang, G. Jiang, J. H. Wu, and Y. Y. Lu, "Numerical solution of nonlinear stochastic Itô-Volterra integral equations by block pulse functions," Mathematica Applicata, vol. 32, no. 4, pp. 935-946, 2019, in Chinese.

[7] J. H. Wu, G. Jiang, and X. Y. Sang, "Numerical solution of nonlinear stochastic Itô-Volterra integral equations based on Haar wavelets," Advances in Difference Equations, vol. 503, pp. 1-12, 2019. 
[8] B. Hashemi, M. Khodabin, and K. Maleknejad, "Numerical solution based on hat functions for solving nonlinear stochastic Itô Volterra integral equations driven by fractional Brownian motion," Mediterranean Journal of Mathematics, vol. 14, no. 24, pp. 1-15, 2017.

[9] J. Guerra and D. Nualart, "Stochastic differential equations driven by fractional Brownian motion and standard Brownian motion," Stochastic Analysis and Applications, vol. 26, no. 5, pp. 1053-1075, 2008.

[10] Y. Mishura and G. Shevchenko, "The rate of convergence for Euler approximations of solutions of stochastic differential equations driven by fractional Brownian motion," Stochastics, vol. 80 , no. 5, pp. 489-511, 2008.

[11] Y. S. Mishura and A. V. Shvaĭ, "An estimate of the rate of convergence of an approximating scheme applied to a stochastic differential equation with an additional parameter," Theory of Probability and Mathematical Statistics, vol. 82, no. 82, pp. 75-85, 2011.

[12] R. Ezzati, M. Khodabin, and Z. Sadati, "Numerical implementation of stochastic operational matrix driven by a fractional brownian motion for solving a stochastic differential equation," Abstract and Applied Analysis, vol. 2014, Article ID 523163, 11 pages, 2014.

[13] F. Mirzaee, Y. Ordokhani, and N. Samadyar, "Hybrid Taylor and block-pulse functions operational matrix algorithm and its application to obtain the approximate solution of stochastic evolution equation driven by fractional Brownian motion," Communications in Nonlinear Science and $\mathrm{Nu}$ merical Simulation, vol. 90, Article ID 105346, 2020.

[14] F. Biagini, Y. Hu, B. Øksendal, and T. Zhang, Stochastic Calculus for Fractional Brownian Motion and Applicationa, Springer, Berlin, Germany, 2008.

[15] Y. S. Mishura, Stochastic Calculus for Fractional Brownian Motion and Related Processes, Springer, Berlin, Germany, 2008.

[16] L. Decreusefond and A. S. üstünel, "Stochastic analysis of the fractional Brownian motion," Potential Analysis, vol. 10, no. 2, pp. 177-214, 1999.

[17] M. Zähle, "Integration with respect to fractal functions and stochastic calculus. I," Probability Theory and Related Fields, vol. 111 , no. 3, pp. 333-374, 1998.

[18] D. Nualart and A. Răşcanu, "Differential equations driven by fractional brownian motion," Collectanea Mathematica, vol. 53, no. 1, pp. 55-81, 2002.

[19] B. Pei and Y. Xu, "Stochastic averaging for stochastic differential equations driven by fractional Brownian motion and standard Brownian motion," Applied Mathematics Letters, vol. 100, Article ID 106006, 2020.

[20] Y. Z. Hu, Y. Liu, and D. Nualart, "Rate of convergence and asymptotic error distribution of euler approximation schemes for fractional diffusions," Annals of Applied Probability An Official Journal of the Institute of Mathematical Statistics, vol. 26, no. 2, pp. 1147-1207, 2016.

[21] J. L. Hong, C. Y. Huang, and X. Wang, "Optimal rate of convergence for two classes of schemes to stochastic differential equations driven by fractional Brownian motions," IMA Journal of Numerical Analysis, vol. 41, no. 2, pp. 1608-1638, 2021. 